\title{
THE TRANSMISSION OF TIDAL WAVES IN ELASTIC ARTESIAN BASINS ${ }^{1}$ )
}

\author{
J. WESSELING \\ Institute for Soil and Water Management Research, \\ Wageningen, The Netherlands
}

\begin{abstract}
SUMMARY
The derivation of the fundamental differential equation for the transmission of tidal waves in elastic artesian beds is given. Starting with the work of STEGGewentz (1933) the general solution of this equation is worked out. A comparison is made between this solution and the transmission of tidal waves in a rigid aquifer and a somewhat different solution obtained by Boscr (1951). A discussion is given on the application of the various solutions of the calculation of hydrological constants of the soil from measurements of waterpressure in the aquifer. From this it is clear that a fairly good approximation of the vertical resistance of the overlying beds may be obtained, without knowledge of the compressibility of the aquifer and the water confined within it. Futhermore the storage capacity of the overlying beds needs not exactly to be known. The values for the vertical resistance, obtained with the described equations, are nearly the same as those found in the special case described by Boscr, but a large difference is present with the values obtained from the equations governing the transmission of tidal waves in rigid artesian beds.

Finally the error of the vertical resistance, introduced through an error in the value of the transmissibility $k D$ of the aquifer, is approximately the same as the error in $k D$.
\end{abstract}

\section{INTRODUCTION}

Consider an homogeneous artesian basin with uniform thickness $D$ and hydraulic conductivity $k$ which is infinite in areal extent (see fig. 1). The aquifer is overlain by a layer with thickness $\left.D^{\prime}{ }^{2}\right)$ and hydraulic conductivity $k^{\prime}\left(k^{\prime} \ll k\right)$. Variations in hydrostatic pressure caused by the tides in a neighbouring river, lake or sea give lateral displacement of water in the aquifer. As a result of the inevitable loss of head accompanying this movement, a reduction in magnitude dependent on the distance from the river, lake or sea of the fluctuations occurs.

Because of the low permeability, lateral movement of water in the upper layer can be ignored. The influence of the tides on the water-level in neighbouring wells reaching into the rigid artesian basin has been dealt with in detail by Stegcewentz (1933). For the fundamental equation governing the case of fig. 1 this author gives

$$
\frac{\delta h}{\delta t}=a^{2} \frac{\delta^{2} \varphi}{\delta x^{2}}
$$

where

$$
a^{2}=\frac{k D}{\mu}
$$

1) Received for publication November 4, 1958.

2) In fact the upper soil layer must be taken from its lower boundary upto the water table. The fluctuations of the water level in this layer are smaller, however, and generally the water table is close to the soil surface. 


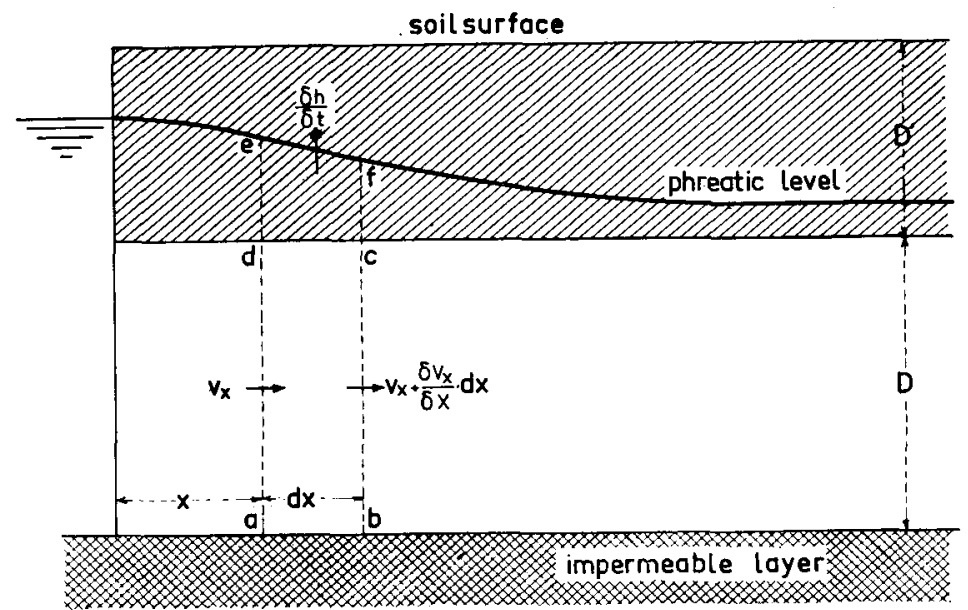

Fig. 1 System SHOWING THE TRANSMission OF TIDAL Waves IN AN AQUIFER REFERRED to by Steggewentz (1933) and USEd IN THE DERIVATION OF THE FUNDaMENTAL DIFFERENTIAL EQUATION.

$h=$ the hydrostatic pressure of the water in the upper layer expressed in meters watercolumn

$t=$ time

$\varphi=$ the hydrostatic pressure of the water in the aquifer, i.e. the water-level in a well penetrating into this layer

$x=$ the horizontal distance from river, lake or sea

$\mu=$ the effective porosity or storage capacity of the upper layer, i.e. the volume of water taken up by unit volume of the soil when water pressure rises unity.

From Darcy's law for the vertical flow in the upper layer follows

$$
\mu \frac{\delta h}{\delta t}=k^{\prime} \frac{\varphi-h}{D^{\prime}}
$$

The term $D^{\prime} / k^{\prime}=c$ is called the vertical resistance of the upper layer. Assuming $C=\mu . c$, the relation between $\varphi$ and $h$ becomes

$$
\varphi=h+C \frac{\delta h}{\delta t}
$$

With the aid of eq. 3 Strogewentz derived the general solution of eq. 1 , this being

$$
\varphi=M+U e^{\alpha x} \sin (n t-\beta x)
$$

in which

$$
\alpha=\frac{n}{2 a^{2}} \sqrt{\frac{\sqrt{1+C^{2} n^{2}+C n+C n}}{1+C^{2} n^{2}}}
$$




$$
\beta=\frac{n}{2 a^{2}} \sqrt{\frac{\sqrt{1+C^{2} n^{2}+C n+C n}}{1+C^{2} n^{2}}}
$$

and

$M=$ the average level of the river or sea

$U=$ amplitude of the tides

$n=\frac{2 \pi}{T}, T$ being the period of the tide

$\alpha=$ constant

$\beta=$ constant.

According to eq. 4a the fluctuations of the river, lake or sea are reduced by a factor $e^{-\alpha x}$ at a distance $x$ from the tidal basin and at this place a phaseshift $\beta x$ occurs.

From $(4 \mathrm{~b})$ and $(4 \mathrm{c})$ it follows that

$$
\begin{aligned}
\frac{\alpha^{2}+\beta^{2}}{2 \alpha \beta} & =\frac{n}{a^{2}}=\frac{n \mu}{k D} \\
1+C^{2} n^{2} & =\frac{n}{a^{2}} \cdot \frac{1}{2 \alpha \beta}
\end{aligned}
$$

If one of the three hydrological constants $\mu, c$ and $k D$ and $\alpha$ and $\beta$ are known, the other two constants may be calculated from (5a) and (5b). Usually the constant $k D$ is determined by means of a pumping test, while $\alpha$ and $\beta$ are determined from observations of the water pressure in the aquifer at various distances from the tidal basin. This method is frequently used to get data about the hydrological constants of the soil which are necessary when designing water supply- and drainage projects (StEGGEwentz, 1933, Trmmers, 1955).

In the above mentioned theory, however, the compressibility of water and the change in volume of the aquifer due to compression of the solid skeleton have not been taken into account. This may lead to completely wrong values of $\mu$ and $c$ (van Hoorn, 1954, Bosch, 1951) and considerable errors may be expected in the values of the computed hydrological constants.

Taking into account these compressibilities, Bosch (1951) derived the fundamental differential equation for the case given in fig. 2 in which a constant phreatic level $\varphi_{P}$ (polder level) is maintained in the bed overlying the aquifer. This differential equation is

$$
\frac{\delta^{2} \varphi}{\delta x^{2}}-\frac{\varphi-\varphi_{P}}{k D c}-\frac{S_{1}}{k D} \cdot \frac{\delta \varphi}{\delta t}=0
$$

in which $S_{1}$ is a dimensionless coefficient of storage having the value (cf. JACOB, 1940):

$$
\mathrm{S}_{1}=\varrho g D\left\{\begin{array}{c}
\varepsilon \\
E_{w}
\end{array}+\frac{1}{E_{s}}\right\}
$$




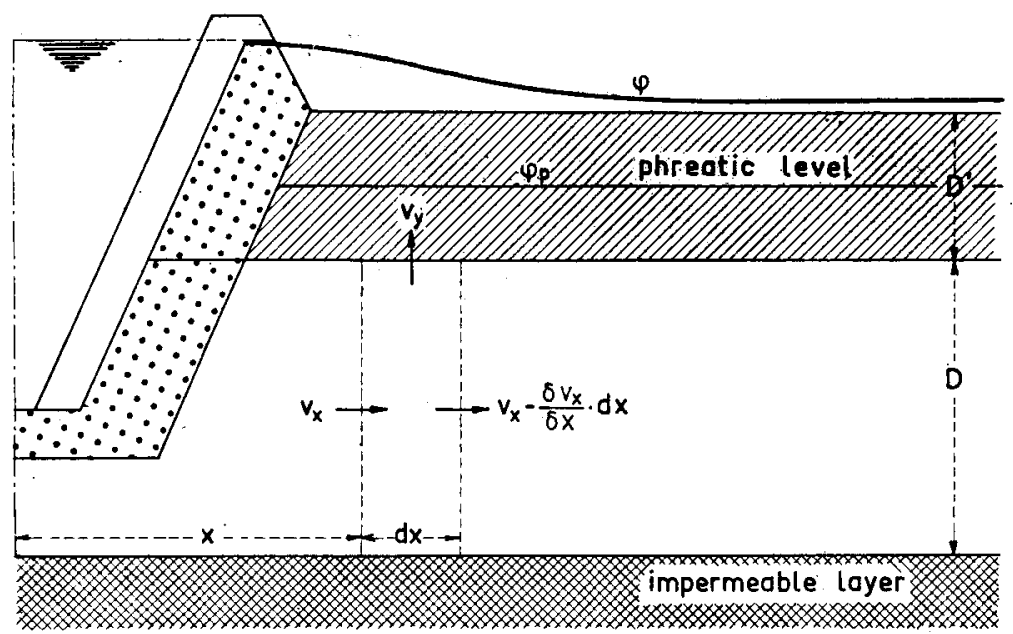

Fig. 2 SYSTEM SHOWING THE TRANSMISSION OF TDAL WAVES IN AN ELASTIC AQUiFER REFERRED TO BX Bosch (1951).

where

$\varrho=$ density of water

$g=$ acceleration of gravity

$\varepsilon=$ porosity of the aquifer

$E_{w}=$ bulk modulus of elasticity of water

$E=$ modulus of compression of the solid skeleton of the aquifer.

Bosch (1951) assumed the general solution of eq. 6 as being

$$
\varphi=\varphi_{p}+\varphi_{0} e^{-\alpha x} \sin (n t-\beta x)
$$

Differentiating this equation with respect to $x$ and $t$, and substitution in eq. 6 yields the conditions

$$
\begin{gathered}
\alpha^{2}-\beta^{2}=\frac{1}{k D c} \\
2 \alpha \beta=\frac{S_{1} n}{k D}-
\end{gathered}
$$

With known $\alpha, \beta$ and $k D$, the values of $c$ and $S_{1}$ may be calculated. Generally the phreatic level in the upper layer is not at a constant height and eqs. 8a and $8 \mathrm{~b}$ are therefore only valid in special cases. Using the method of STEGGEWENTZ a general solution for an elastic aquifer can be obtained, however, for the case under discussion. This solution will be given in detail in the next sections.

\section{The fundamental DifFerential EQUation}

In the solution of Boscr (1951) only the influence of variations in $\varphi$ on the compressibility of the aquifer has been taken into account. Neglecting changes in volume of the upper layer, that will be small compared to those of the aquifer because $D^{\prime} \ll D$ the influence of the fluctuations of $h$ on the compressibility of the aquifer can be introduced by taking a second dimensionless coefficient of storage $S_{2}$ where (ZiJLsTrA, 1957). 


$$
S_{2}=\varrho g D \frac{\mu}{E_{s}}
$$

For the derivation of $S_{2}$ a method similar to that of $\mathrm{J}_{A C O B}(1940)$ and Bosch (1951) was used.

Introducing the coefficients of storage $S_{1}$ and $S_{2}$, the change in mass of water in the element $a b$ ef of fig. 1 will be

$$
\frac{d m}{d t}=\varrho S_{1} d x \frac{d \varphi}{d t}-\varrho S_{2} d x \frac{d h}{d t}
$$

which must be equal to the difference between inflow and outflow into and out of the element. Taking $v_{x}=-k \frac{d \varphi}{d x}$ and $v_{y}=k^{\prime} \frac{\varphi-h}{D^{\prime}}$ the fundamental differential equation becomes

$$
\frac{\delta^{2} \varphi}{\delta x^{2}}-\frac{\varphi-h}{k D c}-\frac{S_{1}}{k D} \frac{\delta \varphi}{\delta t}-\frac{S_{2}}{k D} \frac{\delta h}{\delta t}=0
$$

Differentiating eq. 3, giving the relation between $\varphi$ and $h$ with respect to $t$ and substituting in (11), yields :

$$
\frac{\delta^{2} \varphi}{\delta x^{2}}-A \frac{\delta h}{\delta t}-B \frac{\delta^{2} h}{\delta t^{2}}=0
$$

where

$$
\left.\begin{array}{l}
A=-\frac{1}{k D}\left(\mu+S_{1}+S_{2}\right) \\
B=\frac{k D}{C} \cdot S_{1} \\
C=\mu c
\end{array}\right\}
$$

3 Solution of the FUNDAMENTAL DIFFERENTIAL EQUATION

The solution of the problem under discussion can most easily be obtained by solving the differential equation in terms of $h$. The boundary conditions are :

$$
\begin{array}{ll}
x=0 & \varphi=M+U \sin n t \\
x=\backsim & \frac{\delta \varphi}{\delta t}=0
\end{array}
$$

Substituting the first of these boundary conditions in (3) and integrating, yields :

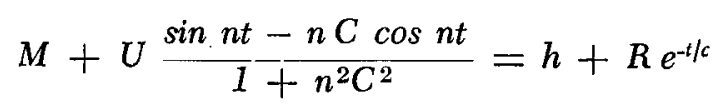

$R$ being the constant of integration.

When dynamic equilibrium has been established, $t$ is very large so the last member of (15) may be neglected (StEGGEWENTZ, 1933) and the following boundary conditions can be taken: 


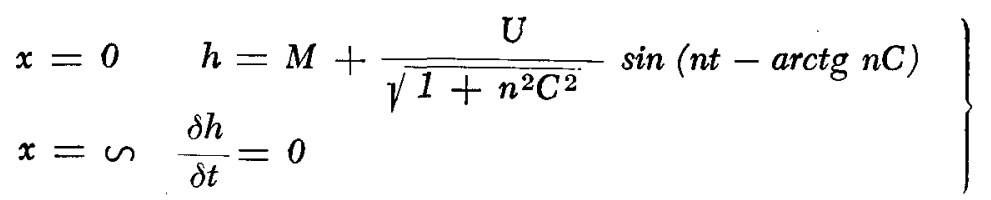

A fundamental equation in terms of $h$ instead of in terms of $h$ and $\varphi$ may be obtained by differentiating (3) with respect to $x$ and substituting the result into eq. 12. The result is given by :

$$
\frac{\delta^{2} h}{\delta x^{2}}+C \frac{\delta^{3} h}{\delta x^{2} \delta t}-A \frac{\delta h}{\delta t}-B \frac{\delta^{2} h}{\delta t^{2}}=0
$$

The problem now has been reduced to the general equation (17) subject to the boundary conditions (16).

The solution of (17) can be represented by the general equation

$$
h=M+I\left[\psi(x) e^{n i t}\right]
$$

where the symbol $I$ means " $-i$ times the imaginary part of " and $\psi$ is a function of $x$ alone.

Substituting this value into eq. 17 gives for the condition which $\psi$ must satisfy

$$
\psi^{\prime \prime}(1+C n i)=\psi\left(A n i-B n^{2}\right)
$$

where $\psi$ " means the second derivate of $\psi$ with respect to $x$.

If the solution of eq. (19) is written in the form

then must satisfy the condition :

$$
\psi=R e^{v x}
$$

$$
v= \pm \sqrt{\frac{(A C-B) n^{2}+n\left(A+B C n^{2}\right) i}{1+C^{2} n^{2}}}
$$

For $x \rightarrow \sim$ the term $e \stackrel{\sqrt{\ldots x}}{\rightarrow} \backsim$ and the plus sign of eq. 38 is not in accordance with the boundary condition $\frac{\delta h}{\delta t}=0$ for $x=\backsim$. The minus sign of (38) gives therefore a solution only.

In order to trace the real and imaginary parts of eq. 21 we take

$$
v=-(\alpha+\beta i)
$$

The real and imaginary parts of (21) now must be equal to respectively the real and imaginary parts of $(22)$ :

$$
\begin{aligned}
& \alpha^{2}-\beta^{2}=\frac{(A C-B) n^{2}}{1+C^{2} n^{2}}=P \\
& 2 \alpha \beta=\frac{n\left(A+B C n^{2}\right)}{1+C^{2} n^{2}}=Q
\end{aligned}
$$

Substituting now eqs. (22) and (20) into the general solution given by (18) yields

$$
h=M+R e^{\alpha x} \sin (n t-\beta x)
$$


The general solution for $h$ from (16) and (25) will then be

$$
h=M+\frac{U}{\sqrt{1+n^{2} C^{2}}} e^{-\alpha x} \sin (n t-\beta x-\operatorname{arctg} n C)
$$

In order to get the solution for $\varphi$ eq. 26 can be substituted into eq. (3). This yields

$$
\varphi=M+\frac{U}{\sqrt{1+n^{2} C^{2}}} e^{-\alpha x}\{\sin (n t-\beta x-\operatorname{arctg} n C)+n C \cos (n t-\beta x \operatorname{arctg} n C)\}
$$

which is identical with

$$
\varphi=M+U e^{-\alpha x} \sin (n t-\beta x)
$$

Eq. 27 is the same as eq. 4a derived by Stegcewentz (1933). The relation between $\alpha$ and $\beta$ and the properties of the artesion basin is given by (23) and (24) in which the compressibility of the aquifer and the water confined within it, are accounted for by eq. 12a.

Neglecting the influence of the fluctuations of $h$ on the compression of the aquifer, $S_{2}$ will be zero (cf. eqs. 3 and 12a) and the fundamental differential equation will be

$$
\frac{\delta^{2} \varphi}{\delta x^{2}}-\frac{\varphi-h}{k D c}-\frac{S_{1}}{k D} \frac{\delta \varphi}{\delta t}=0
$$

The solution of this equation is identical with eq. 27 with the restriction that for $A$ is taken eq. 12 a with $S_{2}=0$.

\section{Discussion}

As is pointed out in the introduction, the equations describing the tidal movement in artesian basins are used to derive values for the storage capacity $\mu$, the vertical resistance $c$ and the transmissibility $k D$ of the basin.

If eq. 4 is applied, $\mu$ and $c$ may be derived if $k D, \alpha$ and $\beta$ are known. Otherwise, $k D$ may be derived from $\alpha, \beta$ and $\mu$ or $\alpha, \beta$ and $c$. The constants $\alpha$ and $\beta$ can easily be determined from water table measurements in water gauge tubes.

Using eq. 8 instead of the storage capacity $\mu$ the storage coefficient $S_{1}$ is obtained. Here again three variables, namely $S_{1}, c$ and $k D$, occur in the equations, $S_{1}$ containing now $D, \varepsilon, E_{w}$ and $E_{s}$. Generally the last mentioned constants are unknown, but from eq. 8c, vallues of $S_{1}$ may be obtained directly.

Using the equations (23) and (24), the constants $A$ and $B$ are introduced besides $k D, \mu$ and $c$. The constants $A$ and $B$ (given by eq. 12a) contain the terms $S_{1}$ and $S_{2}$ which are functions of $D, \varepsilon, \mu, E_{w}$ and $E_{s}$. In order to get a simplified relation between $\mu$ and $c$ the constants $A$ and $B$ may be solved from (23) and (24). This leads to the relation

$$
\begin{aligned}
& A=\frac{Q}{n}+P C \\
& B=\frac{Q C}{n}-\frac{P}{n^{2}}
\end{aligned}
$$


Substituting now the values of $S_{1}$ and $S_{2}$, given by (7) and (9) into eq. 12a, values of $A$ and $B$ expressed in $\mu, c, k, k D, \varrho, g, \varepsilon, E_{w}$ and $E_{s}$ will be obtained. By setting equal these values to those of eq. (29) and (30), two expressions for the permeability $k$ can be obtained which must be equal. After rearranging we get the relation

$\left(\frac{n^{2} \mu^{2} c}{k D}-n^{2} \mu^{2} c^{2} P-P\right)\left(\varepsilon+E_{w} / E_{s}\right)+\left(n \mu^{2} Q c-\mu P\right) E_{w} / E_{s}=0$

When the value of $k D$ is known, the relation between $\mu$ and $c$ may be obtained from eq. 31. For this purpose water table measurements from vaN HoorN (1957) along the river Rhine are used. These measurements were made for the building of a dam near Hagesteijn. For the calculations, the tidal wave of September 11, 1956 has been taken, for which

$$
\begin{gathered}
\alpha=3.33 \times 10^{-3} \\
\beta=2.50 \times 10^{-3} \\
n=12.14 \mathrm{rad} / 24 \mathrm{hrs}
\end{gathered}
$$

The transmissibility $k D$ of $1400 \mathrm{~m}^{2} /$ day was determined by means of a pumping test carried out by the "Rijksinstituut voor Drinkwatervoorziening" at The Hague.

Taking $\varepsilon=0.35$ in eq. 53, $\mu$ and $c$ can be derived for various values of the ratio $E_{w} / E_{s}$ by assuming a certain value of $\mu$ and solving the remaining equation in $c$. The roots of this equation will be either distinct, equal or imaginary. It appears that the minimum value of $\mu$ obeying eq. 31 , may be derived if the determinant of the second order equation in $c$ is zero. If $E_{w} / E_{s}=5$, the minimum value of $\mu=1.116 \times 10^{-3}$ for the tidal wave of Sept. 11, 1956. The corresponding value of $c$ is then 73.9. For values of $\mu$ $<1.116 \times 10^{-3}$, the roots of eq. 31 will be imaginary. For values of $\mu>1.116$ $\times 10^{-3}$ eq. 31 gives two values of $c$. According to eq. 30 the values of $c$ must be such, that $C>P / Q n$ since $B$ has a positive value. Generally one of the two roots of eq. 31 will not obey this condition and for an assumed value of $\mu>1.16 \times 10^{-3}$, only one value of $c$ is obtained. The results of these calculations are given in table 1.

Table 1 Values of $\mu$ and $c$ obeying eq. 30 for various ratios of $E_{\omega} / E_{s}$.

\begin{tabular}{r|c|c|c|c|c|c}
\hline \multicolumn{1}{r|}{$\boldsymbol{E}_{v /} / \boldsymbol{E}_{s}$} & $\frac{1}{10}$ & $\frac{1}{5}$ & 1 & 5 & 10 & 15 \\
\hline $2.10^{-1}$ & 147.67 & 147.73 & 147.82 & 147.87 & & \\
$10^{-1}$ & 147.67 & 147.71 & 147.82 & 147.87 & 147.88 & 147.88 \\
$10^{-2}$ & 147.21 & 147.26 & 147.31 & 147.41 & 147.43 & 147.43 \\
$5.10^{-3}$ & 145.58 & 145.86 & 145.99 & 145.99 & - & - \\
$4.10^{-3}$ & 144.75 & 144.78 & 144.83 & 144.94 & - & - \\
$3.10^{-3}$ & 141.94 & 142.42 & 142.52 & 142.58 & - & - \\
$2.10^{-3}$ & 135.12 & 135.16 & 135.26 & 135.32 & - & - \\
\hline
\end{tabular}

From this table it is clear that the value of $\mu$ has little or no influence on the calculated value of $c$, if $\mu$ is not too small. In this manner a good, approximate, value of $c$ may therefore be obtained in the case of moderately large and large values of $\mu$. In order to obtain the exact value of $c, \mu$ has to 
be known. On the other hand, the value of $\mu$ cannot be obtained from eq. 31 when the value of $c$ is not known exactly.

It appears further from table 1 , that the effect of the ratio $E_{w} / E_{s}$ on the calculated value of $c$ is very small.

In table $1, \varepsilon$ is taken 0.35 . In order to show the influence of $\varepsilon$ on $c$, the ratio $E_{w} / E_{s}=\gamma$ was introduced in eq. 30 . This equation then can be written in the form

$$
\frac{n^{2} \mu^{2} c}{k D}-n^{2} \mu^{2} c P-P+\left(n \mu^{2} Q c-\mu P\right) \frac{\gamma}{\varepsilon+\gamma}=0
$$

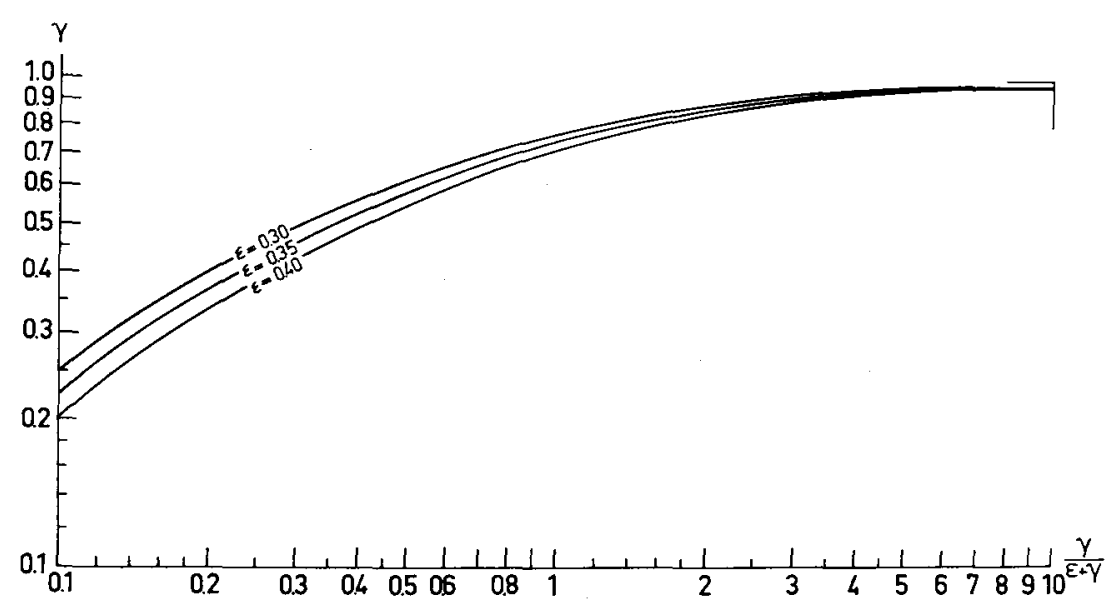

Fig. 3 The RELATION BETWEen $y\left(=E_{w} / E_{s}\right)$ and $\frac{\gamma}{\varepsilon+\gamma}$ For various values of the
porosity $\varepsilon$ OF THE AQUifER.

The values of $\mu$ and $c$ are now only dependent on the last term of this equation. Generally $\varepsilon$ ranges from 0.3 to 0.4 for artesian basins. The relation between $\gamma$ and $\frac{\gamma}{\varepsilon+\gamma}$, for $\varepsilon$ is $0.3,0.35$ and 0.40 , is given in fig. 3. The range of $\frac{\gamma}{\varepsilon+\gamma}$ in this figure is almost completely covered by table 1. Therefore the influence of $\varepsilon$ is also very small.

The term $\frac{\gamma}{\varepsilon+\gamma}=\frac{1}{\mu} \frac{S_{2}}{S_{1}}$. This can be derived from eqs. 7 and 9 . With increasing $\gamma$ the ratio $\frac{S_{2}}{S_{1}}$ increases at the same rate. On the other hand this ratio is highly dependent on the value of $\mu$ as could be expected from the theory.

The last variable occuring in eq. 31 is the transmissibility $k D$. In order to investigate the influence of this factor we differentiate eq. 31 with respect to $c$. Then we get

$$
\frac{d(k D)}{d c}=\frac{1}{k D-k^{2} D^{2}\left(2 P c-\frac{Q}{n} \cdot \frac{\gamma}{\varepsilon+\gamma}\right)}
$$


Substituting the mean values of $k D, c$ and $\frac{\gamma}{\varepsilon+\gamma}$ (being 1400, 147 and 0.93 respectively) in (55) we get

$$
d(k D)=-0,1 d c
$$

The mean value of $k D$ is approximately 10 times the mean value of $c$. This means that if $k D$ is found $10 \%$ too small, the calculated value of $c$ is about $10 \%$ too large.

From table 1 it is obvious, that the ratio $E_{w} / E_{s}=\gamma$ has little or no influence on the relation between $\mu$ and $c$. In order to compare the results of eq. 31 with those of eq. 4 and 8 the ratio $E_{w} / E_{s}=5$ has been taken only. The results are given in table 2.

Table 2 Comparision of the results of eq. 4 (Stegcewentz), 8 (Bosch) and 31 . For the latter case only the results of $E_{w} / E_{s}=5$ are taken.

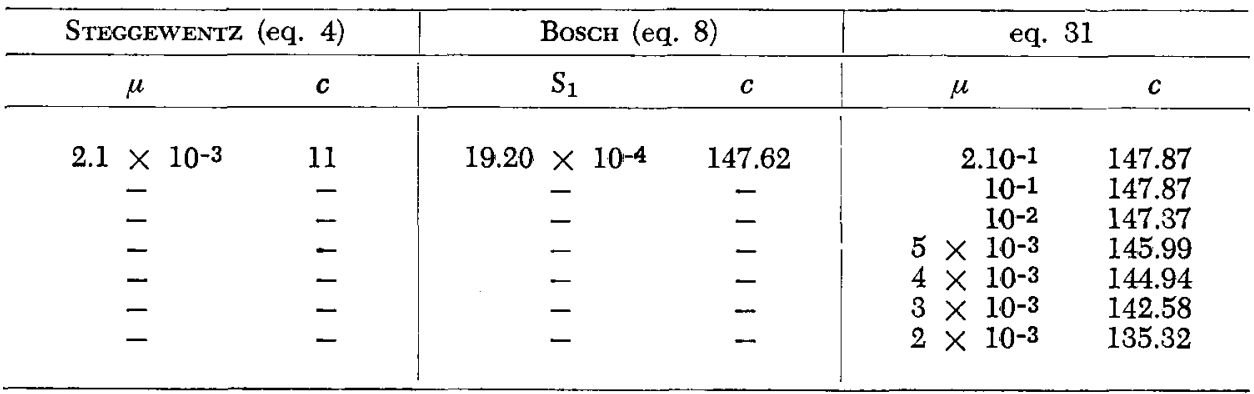

The values of both $\mu$ and $c$ calculated according to eq. 4 , are very small compared with those according eq. 31. The value $c=147$ which implies $k^{\prime}=0.04 \mathrm{~m} /$ day agrees very well with conductivity data obtained from drain outflow measurements in this area carried out by VAN Hoons. In order to compare the results from the equations 8 and 31 , the values of $S_{1}$ and $S_{2}$ will have to be calculated. According to (12a), $S_{1}$ and $S_{2}$ are

$$
\begin{aligned}
S_{1} & =B \frac{k D}{c} \\
S_{1}+S_{2} & =A k D-\mu
\end{aligned}
$$

The values of $A$ and $B$ were computed from eqs. (29) and (30). The calculated values for $S_{1}$ and $S_{2}$ are given in table 3 together with those derived from eq. 8 .

Table 3 Calculated values for $S_{1}$ and $S_{2}$ from eqs. 8 (Воsсн) and 31 . In the latter case only the ratio $E_{w} / E_{s}=5$ has been taken.

\begin{tabular}{c|c|c|c}
\hline Bosch (eq. 8) & \multicolumn{3}{|c}{ eq. 31 } \\
\hline$S_{1}$ & $\mu$ & $S_{1}$ & $S_{2}$ \\
\hline $19.20 .10^{-4}$ & $2.10^{-1}$ & $19.19 \cdot 10^{-4}$ & $3.49 .10^{-4}$ \\
- & $10^{-1}$ & $19.17 .10^{-4}$ & $1.77 .10^{-4}$ \\
- & $10^{-2}$ & $18.89 .10^{-4}$ & $1.15 .10^{-4}$ \\
- & $5.10^{-3}$ & $18.57 .10^{-4}$ & $0.08 .10^{-4}$ \\
- & $4.10^{-3}$ & $18.41 .10^{-4}$ & $0.06 .10^{-4}$ \\
- & $3.10^{-3}$ & $18.13 .10^{-4}$ & $0.04 .10^{-4}$ \\
- & $2.10^{-3}$ & $17.51 .10^{-4}$ & $0.02 .10^{-4}$ \\
\hline
\end{tabular}


From this table it is clear that a fairly good approximation of $c$ can be obtained by using a special solution as given by eq. 8. This is due to the fact that the effect of the fluctuating water level in the overlying bed on the compressibility of the aquifer, is very small.

\section{ACKNOWLEDGEMENT}

The author is indebted to Mr. K. ZiJLstra of the Provinciale Waterstaat Zuid-Holland at The Hague for his personal communication and his criticism on the solution and to Mr. P. Bruin of the Laboratory of Physics and Meteorology of the Landbouwhogeschool Wageningen for his help and advice in solving the problem under discussion.

\section{BIBLIOGRAPHY}

Bosch, H. : Geo-hydrologisch onderzoek Bergambacht. Hydr. Coll. H.C. (S) nr. 255 A. 1951 (typescr.).

Hoors, J. W. vaN : Interimrapporten stuwbouw Hagesteijn nrs. 1, 2 en 3, 1957 (typescript).

$\mathrm{J}_{\mathrm{ACOB}}, \mathrm{C}$. E.: On the flow of water in an elastic artesian aquifer. Trans. Am. Geoph. Union, 1940, 576-586.

StegGeweNTz, J. H. : De invloed van de getijbeweging van zeeën en getijrivieren op de stijghoogte van het grondwater. Thesis Delft 1933.

Timmers, H. J. : Determination of soil permeability in situ. Neth. J. of Agr. Sci. 3 (1955) $119-126$.

Zrjlstra, K. C.: Personal communication 1957. 